\title{
Epidermal differentiation complex (locus 1q21) gene expression in head and neck cancer and normal mucosa
}

\author{
Tomasz Tyszkiewicz ${ }^{1 *}$, Michal Jarzab ${ }^{2 *}$, Cezary Szymczyk ${ }^{3}$, Monika Kowal ${ }^{1}$, \\ Jolanta Krajewska ${ }^{1}$, Magdalena Jaworska ${ }^{4}$, Marcin Fraczek ${ }^{5}$, Anna Krajewska ${ }^{6}$, \\ Ewa Hadas ${ }^{6}$, Michal Swierniak ${ }^{1,9}$, Jaroslaw Markowski ${ }^{7}$, Dariusz Lange, \\ Stanislaw Poltorak ${ }^{3}$, Malgorzata Wiench ${ }^{8}$, Tomasz Krecicki ${ }^{5}$, \\ Jerzy Jarzab $^{6}$, Adam Maciejewski ${ }^{3}$
}

${ }^{1}$ Department of Nuclear Medicine and Endocrine Oncology, Maria Sklodowska-Curie Memorial Cancer Center and Institute of Oncology, Gliwice Branch, Poland

${ }^{2}$ III Department of Radiotherapy and Chemotherapy, Maria Sklodowska-Curie Memorial Cancer Center and Institute of Oncology, Gliwice Branch, Poland

${ }^{3}$ Department of Oncological and Reconstructive Surgery, Maria Sklodowska-Curie Memorial Cancer Center and Institute of Oncology, Gliwice Branch, Poland

${ }^{4}$ Department of Tumor Pathology, Maria Sklodowska-Curie Memorial Cancer Center and Institute of Oncology, Gliwice Branch, Poland

${ }^{5}$ Department of Otolaryngology, Head and Neck Surgery, Wroclaw Medical University, Wroclaw, Poland

${ }^{6}$ Department of Internal Medicine, Dermatology and Allergology, Silesian Medical University, Zabrze, Poland

${ }^{7}$ Department of Laryngology, Silesian Medical University, Katowice, Poland

${ }^{8}$ School of Dentistry, School of Cancer Sciences, University of Birmingham, Birmingham, United Kingdom

${ }^{9}$ Genomic Medicine, Department of General, Transplant and Liver Surgery, Medical University of Warsaw, Poland

\begin{abstract}
Epidermal differentiation complex (EDC) comprises a number of genes associated with human skin diseases including psoriasis, atopic dermatitis and hyperkeratosis. These genes have also been linked to numerous cancers, among them skin, gastric, colorectal, lung, ovarian and renal carcinomas. The involvement of EDC components encoding S100 proteins, small proline-rich proteins (SPRRs) and other genes in the tumorigenesis of head and neck squamous cell cancer (HNSCC) has been previously suggested. The aim of the study was to systematically analyze the expression of EDC components on the transcript level in HNSCC. Tissue specimens from 93 patients with HNC of oral cavity and 87 samples from adjacent or distant grossly normal oral mucosa were analyzed. 48 samples (24 tumor and 24 corresponding surrounding tissue) were hybridized to Affymetrix GeneChip Human 1.0 ST Arrays. For validation by quantitative real-time PCR (QPCR) the total RNA from all
\end{abstract}

Correspondence address: $\mathrm{M}$. Jarzab, MD, PhD

Maria Sklodowska-Curie Memorial Cancer Center

and Institute of Oncology

Wybrzeze Armii Krajowej 15, 44-101 Gliwice, Poland;

tel.: +483227886 17, fax: +48322788441

e-mail: mjarzab@io.gliwice.pl

*Both authors contributed equally to the study 
180 samples collected in the study was analyzed with Real-Time PCR system and fluorescent amplicon specific-probes. Additional set of samples from 14 patients with laryngeal carcinoma previously obtained by HG-U133 Plus 2.0 microarray was also included in the analyses. The expression of analyzed EDC genes was heterogeneous. Two transcripts (S100A1 and S100A4) were significantly down-regulated in oral cancer when compared to normal mucosa ( 0.69 and 0.36 -fold change, respectively), showing an opposite pattern of expression to the remaining S100 genes. Significant up-regulation in tumors was found for S100A11, S100A7, LCE3D, S100A3 and $S 100 A 2$ genes. The increased expression of $S 100 A 7$ was subsequently validated by QPCR, confirming significant differences. The remaining EDC genes, including all encoding SPRR molecules, did not show any differences between oral cancer and normal mucosa. The observed differences were also assessed in the independent set of laryngeal cancer samples, confirming the role of $\$ 100 A 3$ and $L C E 3 D$ transcripts in HNC. In HNC of oral cavity only one family of EDC genes (S100 proteins) showed significant cancer-related differences. A number of other transcripts which showed altered expression in $\mathrm{HNC}$ require further validation. (Folia Histochemica et Cytobiologica 2014, Vol. 52, No. 2, 79-89)

Key words: HNSCC; oral SCC; laryngeal cancer; epidermal differentiation complex; S100 genes; LCE3D; gene expression; microarrays; QPCR

\section{Introduction}

Epidermal differentiation complex (EDC) comprises many genes clustered on chromosomal human region lq21 and coding for proteins involved in late differentiation of epithelial cells. These genes are divided into three families encoding: cornified cell envelope (CE) precursors (involucrin, loricrin, small proline-rich proteins - SPRRs and the "late cornified envelope" proteins, LCEs), calcium-binding proteins (S100) with EF-hand domains, and "fused gene" proteins (SFTPs, 'S100 Fused Type proteins') involving filaggrin, filaggrin-2, repetin, trichohyalin, cornulin and hornerin [1]. Epidermal differentiation molecules are important especially for a plethora of skin diseases [1]. They may also constitute an important feature differentiating squamous cell carcinomas (SCC) from other types of cancer, e.g. adenocarcinomas, despite the fact that the epidermal differentiation is also an important mechanism in adenocarcinoma [2].

More than 10 years ago Zucchini et al. provided an early insight into the expression profile of EDC genes in normal and cancer cells by DNA macroarray [3]. The largest and best known family of EDC genes are these which encode S100 proteins. 21 of human S100 genes are clustered at chromosomal region frequently deleted, translocated or duplicated in epithelial and soft-tissue tumors [4]. It is believed that each member of the gene S100 family plays a role in tumorigenesis and mechanisms of metastases, however, their precise role in the development and promotion of cancer is largely unclear. It was shown that S100 proteins were involved in tumor metastasis by interacting with matrix metalloproteinases, cytoskeletal proteins, p53, Jab 1, Cox-2 and BRCA1 proteins [5]. S100B, one of the best-studied proteins (located outside EDC complex on chromosome 21q22.3), is currently used as a biomarker for melanoma, reflecting tumor bur- den and correlating with overall survival [4]. S1004A protein is considered another potential key player in tumor progression and metastasis [6, 7]. However, S100 proteins can also play an anti-oncogenic role in head and neck cancer (HNC) [8].

Late cornified envelope (LCE) proteins, belonging to cornified envelope precursors, are encoded by LCE genes organized into three linked subclusters on 1q21. They are expressed primarily in skin and are regulated by UV irradiation and calcium [9]. Their expression is altered in skin diseases - psoriasis and atopic dermatitis [1].

Increasing number of reports suggest the role of S100 proteins [10-12], SPRRs [13] and other EDC genes in $\mathrm{HNC}$, including laryngeal and oral carcinomas. One of the earliest studies of expression profiling in head and neck squamous cell carcinoma (HNSCC) was carried out in hypopharyngeal cancer samples against histologically normal tissue [14]. 1q21 locus overexpression was noted as a clear feature of gene expression in these tumors. Hudson et al. also suggested potential role of EDC complex genes in HNSCC in their microarray study [15]. The aim of our study was to analyze systematically the expression of the EDC genes on the transcript level in head and neck cancer of oral cavity.

\section{Material and methods}

Patients. Tissue specimens were collected retrospectively from 93 patients with $\mathrm{HNC}$ of oral cavity, operated on in the Department of Oncological Surgery, Maria Sklodowska-Curie Memorial Cancer Center and Institute of Oncology (Gliwice, Poland) in years 2007-2012. Intraoperative tumor specimens were available from all patients. Additionally, 87 samples from adjacent or distant grossly normal oral mucosa were collected (1-3 per patient). The study was approved by a local ethics committee and all patients gave the informed consent. 
Table 1. QPCR amplicon design

\begin{tabular}{|l|c|c|c|c|}
\hline Gene & Fluorescent probe sequence & Forward primer sequence & Reverse primer sequence & Efficiency \\
\hline$S 100 A 7$ & cttccca & ctgctgacgatgatgaagga & cgaggtaatttgtgcccttt & 1.86 \\
\hline$S 100 A 9$ & gacctgga & gtgcgaaaagatctgcaaaa & tcagctgcttgtctgcattt & 1.81 \\
\hline$S P R R 1 A$ & ctccagca & caccccaaagtgcctgag & ttctgcttggtcttctgctg & 1.80 \\
\hline$S P R R 1 B$ & cagctccc & gagagacttaagatgaaagcaaatga & tgaaagtgaatttaatgggggta & 1.86 \\
\hline
\end{tabular}

Amplicons were designed with Universal Probe Library locked-nucleic acid probes, as described in Methods section

Among 24 subjects in whom the detailed microarray examination was carried out there were $79 \%$ of male patients, median age 57 years. In that group there were $4 \%$ of patients with grade 1 tumor, $66 \%$ G2 and $30 \%$ G3 subjects.

Histopathological assessments were carried out in a typical manner: specimens were fixed in $10 \%$ buffered formalin and embedded in paraffin blocks, cut into ultra-thin paraffin sections 3-4 $\mu \mathrm{m}$ thick and stained with hematoxylin and eosin (HE). Tissue fragments, weighting about $70 \mu \mathrm{g}$, adjacent to fragments sent for histological examination were placed on dry ice immediately after collection and stored deep frozen at $-80^{\circ} \mathrm{C}$.

Additionally, data from 14 patients with squamous cell laryngeal carcinoma, diagnosed and surgically treated between 2005 and 2007 in the Laryngology Department of the Silesian Medical University in Katowice, Poland, previously assessed in our laboratory by microarray profiling (as described by Markowski et al., 2009), were included in the analysis [16].

RNA isolation. RNA was extracted from fresh frozen tissues using a standard RNeasy Micro Kit protocol including a digestion step with RNase-free DNase I (Qiagen). The quantity and quality control was performed using NanoDrop 1000 spectrophotometer (Thermo Scientific) and RNA 6000 Nano LabChip kit (Agilent Technologies). Total RNAs RIN values were between 6.8 and 9.5 .

Microarray analysis of gene expression. $500 \mathrm{ng}$ of total RNA from 48 tissue fragments (24 tumor and 24 corresponding surrounding tissues) were used for the microarray experiment (Affymetrix GeneChip Human 1.0 ST Arrays). The cDNA was synthesized, fragmented and labeled with biotin using the Ambion WT Expression Kit (Life Technologies) following the procedure described in the Ambion WT Expression Protocol. The arrays were incubated in a GeneChip Hybridization Oven 640 (Affymetrix), washed and stained on a GeneChip Fluidics Station 450 (Affymetrix) using the Hybridization Wash and Stain Kit (Affymetrix) and the Fluidics Procedure FS450_0007. The arrays were processed with a GeneChip Scanner 3000 (Affymetrix).

For laryngeal cancer samples, older generation high density microarrays, Human Genome U 133 Plus 2.0 (Affymetrix) were used.
Real-Time Quantitative RT-PCR (QPCR). Total RNA from 180 fresh frozen oral cavity cancer samples (all specimens collected in the study) was used for QPCR validation. Reverse transcription was performed using Qiagen Omniscript RT Kit. The input RNA for each sample was $200 \mathrm{ng}$ in a final volume of $20 \mu \mathrm{L}$. All samples were diluted 10 times prior to QPCR. Reactions were performed in duplicates on 384-well plates using 7900HT Fast Real-Time PCR system (Life Technologies). The QPCR conditions consisted of a first step at $50^{\circ} \mathrm{C}$ ( 2 min; activation and incubation with AmpErase UNG) and $95^{\circ} \mathrm{C}$ (10 min; activation AmpliTaq Gold polymerase), followed by 40 cycles of amplification $\left(95^{\circ} \mathrm{C}, 15 \mathrm{~s} ; 60^{\circ} \mathrm{C}, 1 \mathrm{~min}\right)$. Each reaction contained $10 \mu \mathrm{l}$ of Taqman Universal Mastermix (Life Technologies), $200 \mathrm{nM}$ of each primer, $2.8 \mu \mathrm{l}$ RNasy free water and $5 \mu \mathrm{l}$ of diluted cDNA template. For amplicon design we used the Roche Universal Probe Library (Table 1) following general conditions: 1) an amplified sequence for all transcripts covered by the microarray probe, 2) amplicon unique to human transcriptomic database (confirmed by BLAST), 3) no known single nucleotide polymorphisms (SNPs) in any of primers or probe sequence (confirmed by BLAST SNP). Standard curve was performed for each amplicon of the 8 duplicate concentrations (1000 ng, $500 \mathrm{ng}, 200 \mathrm{ng}, 100 \mathrm{ng}, 40 \mathrm{ng}, 20 \mathrm{ng}$, $8 \mathrm{ng}$, and $2 \mathrm{ng}$ of total RNA). The linear regression slope of the standard curve indicated amplification efficiency. For normalization of QPCR data three transcripts were chosen as reference: EIF3S10, HADHA and UBE2D2, based on previously carried out QPCR analysis of $S P R R$ gene expression in skin [17]. The normalization factor was obtained using the GeNorm applet for Microsoft Excel, based on the three reference genes tested. After quality assessment, 91 tumor samples and 84 samples of normal mucosa were used for final analysis.

Data analysis. Microarray data were pre-processed by RMA algorithm, using Bioconductor 1.5 packages. Expression data were transferred to BRB Array Tools 4.3.2 (developed by Dr. Richard Simon and BRB Array Development Team) and subset of genes located in 1q21 chromosomal region was selected. Among the genes of EDC cluster represented by probe-sets on GeneChip Human 1.0ST microarray there 


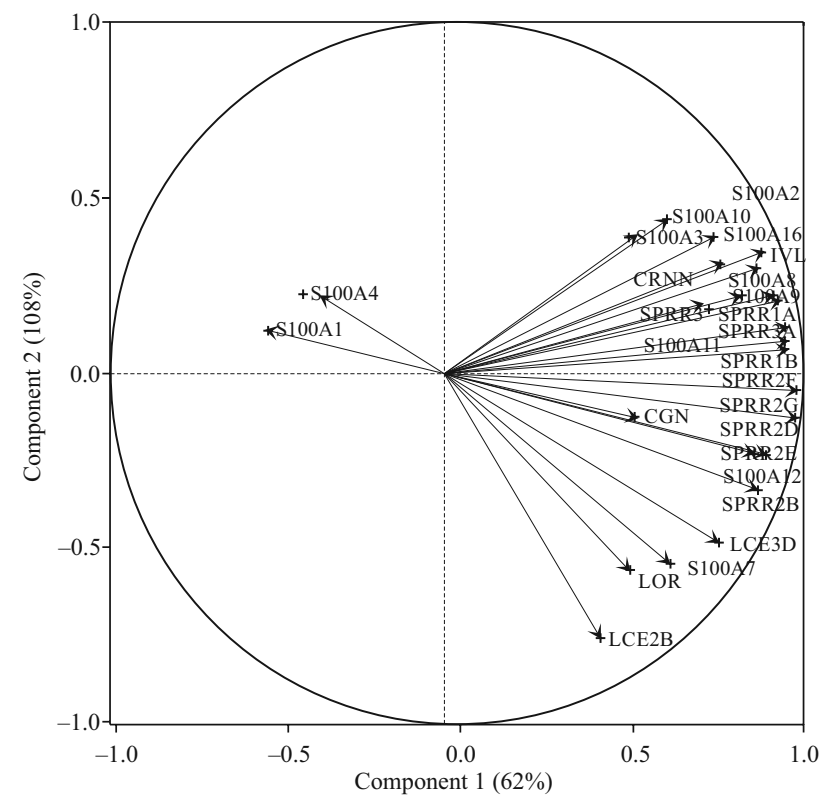

Figure 1. Principal component analysis of gene expression values in the whole dataset or oral cancer specimens and oral mucosa. First two principal components are depicted on axes $\mathrm{X}$ and $\mathrm{Y}$

were the following transcripts: $C G N, L C E 2 B, I V L, S P R$ R1A, SPRR3, SPRR1B, LOR, S100A9, S100A1, S100A10, S100A11, CRNN, LCE3D, SPRR2D, SPRR2B, SPRR2, SPRR2E, SPRR2G, S100A12, S100A8, S100A7, S100A4, $S 100 A 3, S 100 A 2, S 100 A 16, S P R R 2 F$. Within this limited list the statistically significant transcripts were identified by a supervised analysis using random variance t-test with False Discovery Rate estimation according to Benjamini-Hochberg algorithm. False Discovery Rate below 0.1 (10\%) was deemed significant. Microarray data for selected transcripts were also exported out to JMP Genomics to carry on the Principal Component Analysis. Normalized QPCR data were assessed by non-parametric U Mann-Whitney test.

\section{Results}

\section{Analysis of 1q21 epidermal differentiation genes in oral cancer}

Using microarray technology we analyzed expression of all EDC 1q21 genes in the panel of 24 oral SCC and compared the expression to the corresponding normal mucosa specimens from the same individuals. First, an unsupervised Principal Component Analysis (PCA) was carried out on the full dataset. The gene expression patterns within first two principal components are summarized in Figure 1. It can be clearly observed that two transcripts (S100A1 and S100A4) show an opposite pattern of expression to the remaining EDC genes. Both genes are highly significantly down-regulated in oral cancer when compared to adjacent normal mucosa (Figure 2A, Table 2). Both genes show similar level of expression in normal mucosa, while the down-regulation is higher for S100A1 (approx. 3 -fold decrease) in comparison to $S 100 A 4$ (30\% lower expression in cancer). It is to be noted that both genes exhibit lower expression in normal oral mucosa than the remaining S100 genes. The PCA also revealed that four genes: $S 100 A 7$, loricrin and two late cornified envelope genes ( $L C E 2 B$ and $3 D)$ show slightly divergent profile in comparison to the remaining set of relatively uniform transcripts (Figure 1). In microarray analysis, $S 100 A 7$ and $L C E 3 D$ showed significant differences in gene expression (Figure 2B, Table 2), both genes being up-regulated in cancer tissues, while for $L C E 2 B$ and $L O R$ no significant differences were confirmed. Within the remaining genes highly significant differences were also noted for S100A11, while $S 100 A 3, S 100 A 2$ (up-regulated in cancer) and cingulin (slightly down-regulated in cancer) showed a trend towards difference. Up-regulation of $S 100 A 7$ and $L C E 3 D$ was almost fourfold in malignant tissue, while all remaining genes showed $37-57 \%$ increase in comparison to adjacent normal mucosa (Table 2).

\section{Analysis of EDC in an independent set of laryngeal cancer samples}

The expression of EDC genes was analyzed independently in the group of laryngeal cancers $v s$. normal mucosa samples. Three genes found to be changed in oral cancer ( $S 100 A 3, L C E 3 D$ and $C G N)$ similarly showed significant differences in laryngeal cancer, with comparable fold-change ratios (Table 3). All other differentiating genes in oral cancer showed no significant differences in laryngeal cancer set (FDR $>10 \%$ ). Three other transcripts, not differentially expressed in oral cancer, were significantly changed in laryngeal cancer (FDR < 10\%), namely SPRR $2 G$ (up -regulated in cancer more than eight-fold), $C R N N$ and S100A10 (both down-regulated in cancer; Table 3).

\section{Validation by quantitative real time PCR}

For the validation study, we selected $S 100 A 7$, a gene of high significance in oral cancer (ranked \# 3 within S100 transcripts in microarray analysis), to provide a confirmation of positive results and three genes of no significance to provide a confirmation of negative findings in the much larger population. The QPCR results showed a highly significant difference for S100A7 gene while no differences were observed for the remaining genes (Figure 3), confirming the 

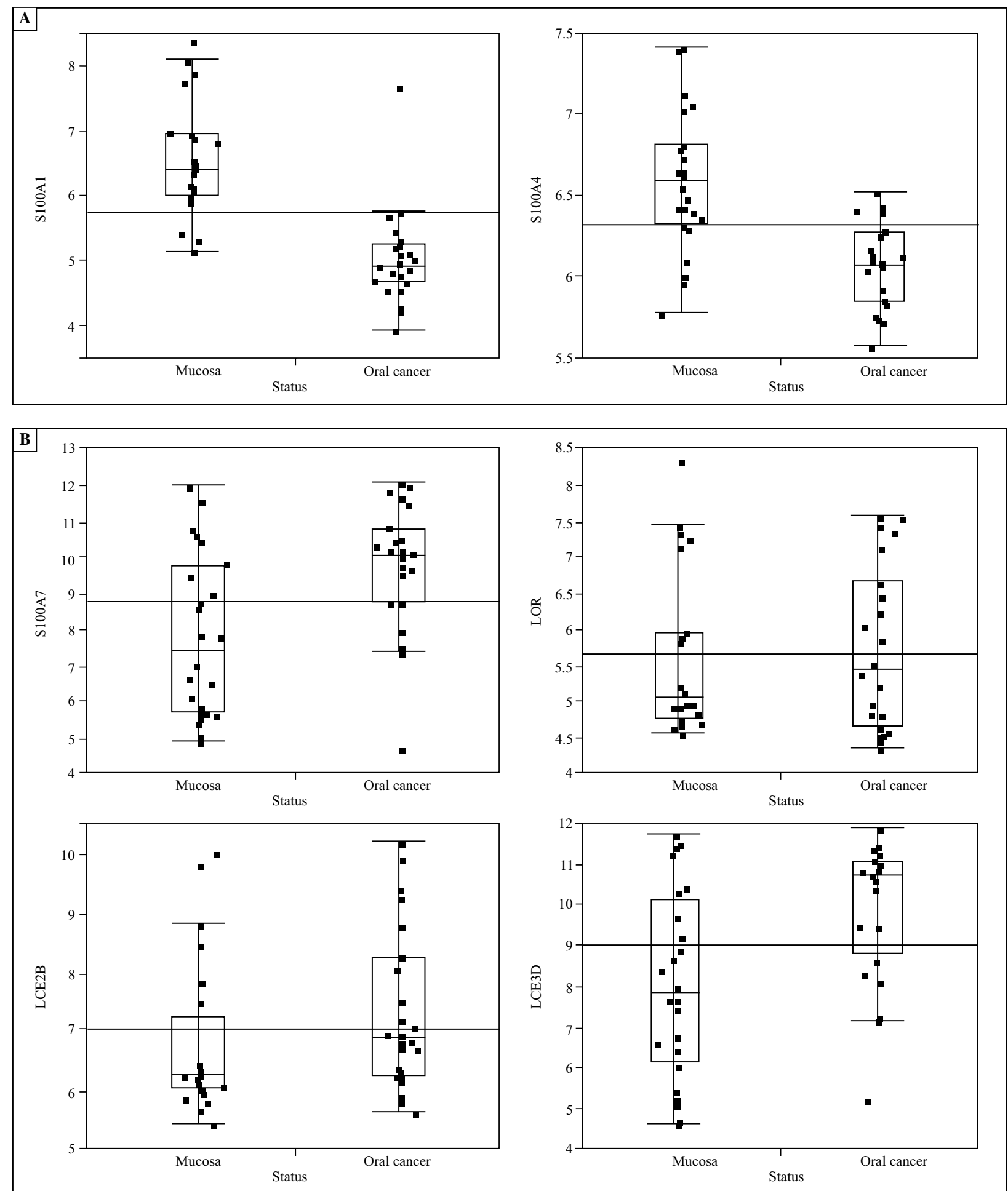

Figure 2. Gene expression measurement by microarray study for selected down-regulated (A) and up-regulated genes (B). Significant differences were observed for S100A1, S100A4, S100A7 and LCE3D (FDR < 5\%). On Y axis the log2-transformed expression of genes from microarray analysis is shown (arbitrary units). Vertical line denotes the grand mean expression value in both groups

significance of our findings using an independent method in much larger dataset. Median of S100A7 expression in normal mucosa group was 0.07 arbitrary units, in oral cancer median was 0.29 units, the ratio of geometric means between tumor and normal samples was 5.4. These results confirmed the difference observed in microarray study (3.9-fold increase in cancer). 
Table 2. Expression of EDC genes in oral cancer (microarray study)

\begin{tabular}{|c|c|c|c|c|}
\hline Symbol or full name of the gene & FDR & Gene expression in mucosa & Gene expression in oral cancer & Fold-change \\
\hline \multicolumn{5}{|l|}{ S100 calcium binding proteins } \\
\hline$S 100 A 1$ & $2.33 \mathrm{E}-06^{* *}$ & 91.95 & 32.84 & 0.36 \\
\hline S100A4 & $4.80 \mathrm{E}-05^{* *}$ & 96.54 & 67.05 & 0.69 \\
\hline S100A7 & $0.0058^{* *}$ & 224.98 & 868.64 & 3.86 \\
\hline S100A11 & $0.00924 * *$ & 2283.07 & 3468.52 & 1.52 \\
\hline$S 100 A 3$ & $0.0559^{*}$ & 59.34 & 81.23 & 1.37 \\
\hline S100A2 & $0.0947^{*}$ & 258.45 & 404.53 & 1.57 \\
\hline S100A12 & 0.162 & 155.3 & 268.64 & 1.73 \\
\hline$S 100 A 10$ & 0.459 & 3359.25 & 3712.7 & 1.11 \\
\hline S100A9 & 0.900 & 2644.01 & 2405.17 & 0.91 \\
\hline S100A16 & 0.975 & 1758.79 & 1776.2 & 1.01 \\
\hline S100A8 & 0.975 & 6329.6 & 6197.08 & 0.98 \\
\hline \multicolumn{5}{|l|}{ Other genes } \\
\hline $\begin{array}{l}L C E 3 D \\
\text { (late cornified envelope 3D) }\end{array}$ & $0.00699 * *$ & 273.69 & 1035.47 & 3.78 \\
\hline$C G N$ (cingulin) & $0.095^{*}$ & 63.01 & 52.76 & 0.84 \\
\hline$C R N N$ (cornulin) & 0.207 & 1896.74 & 779.82 & 0.41 \\
\hline $\begin{array}{l}\angle C E 2 B \\
\text { (late cornified envelope 2B) }\end{array}$ & 0.273 & 109.46 & 159.73 & 1.46 \\
\hline$I V L$ (involucrin) & 0.736 & 441.03 & 525.26 & 1.19 \\
\hline$L O R$ (loricrin) & 0.777 & 48.57 & 54.23 & 1.12 \\
\hline \multicolumn{5}{|l|}{ Small proline-rich proteins } \\
\hline SPRR3 & 0.187 & 3809.31 & 1790.96 & 0.47 \\
\hline$S P R R 2 B$ & 0.404 & 130.63 & 218.72 & 1.67 \\
\hline SPRR1B & 0.408 & 1889.66 & 2716.83 & 1.44 \\
\hline$S P R R 2 E$ & 0.408 & 689.2 & 1129.1 & 1.64 \\
\hline$S P R R 2 G$ & 0.43 & 2023.99 & 2816.68 & 1.39 \\
\hline$S P R R 2 D$ & 0.451 & 58.68 & 79.9 & 1.36 \\
\hline$S P R R 2 F$ & 0.479 & 2297.76 & 3018.76 & 1.31 \\
\hline$S P R R 2 B$ & 0.705 & 232.82 & 319.84 & 1.37 \\
\hline$S P R R 2 A$ & 0.959 & 2718.04 & 2930.41 & 1.08 \\
\hline$S P R R 2 G$ & 0.959 & 1387.42 & 1329.25 & 0.96 \\
\hline SPRR1A & 0.975 & 3402.43 & 3487.3 & 1.02 \\
\hline
\end{tabular}

Gene expression values are presented as geometric means, the fold-change is also presented

*False discovery rate (FDR) was deemed significant if within $1-10 \%(0.01-0.1)$, and **highly significant if FDR < $0.01(1 \%)$

\section{Summary of the results}

In two datasets of head and neck cancer (oral ca and laryngeal ca) we consistently observed an overexpression of three genes - S100A3, LCE3D and $C G N$. Moreover, in oral cancer we found an increased expression of the other S100 transcripts, one of them, $S 100 A 7$, was validated in a larger group of patients by independent method. Two S100 genes - S100A1 and
S100A4 showed highly significant decrease in gene expression in oral cancer. In oral cancer, we found no differences in SPRR genes expression nor other EDC genes, e.g. for involucrin.

\section{Discussion}

In the current study it was the expression of S100 genes which appeared as the most significant tumor- 
Table 3. Expression of EDC genes in laryngeal cancer (microarray study)

\begin{tabular}{|l|c|c|c|c|}
\hline Symbol & FDR & Gene expression in mucosa & Gene expression in laryngeal cancer & Fold-change \\
\hline Up-regulated in cancer & 19.47 & 161.05 & 8.27 \\
\hline$S P R R 2 G$ & 0.00194 & 26.23 & 43.29 & 1.65 \\
\hline$S 100 A 3$ & $0.0713^{\S}$ & 62.43 & 265.26 & 4.25 \\
\hline$L C E 3 D$ & $0.0852^{\S}$ & 54.63 & 30.45 & 0.56 \\
\hline Down-regulated in cancer & $0.0182^{\S}$ & 1533.34 & 96.99 & 0.06 \\
\hline$C G N$ & 0.0198 & 171.53 & 81.96 & 0.48 \\
\hline$C R N N$ & 0.0478 & & & \\
\hline S100A10 &
\end{tabular}

Gene expression values are presented as geometric mean values. Only transcripts with FDR $<0.1(10 \%)$ are shown

${ }^{\S}$ Results significant also in the oral cancer microarray analysis (Table 2)
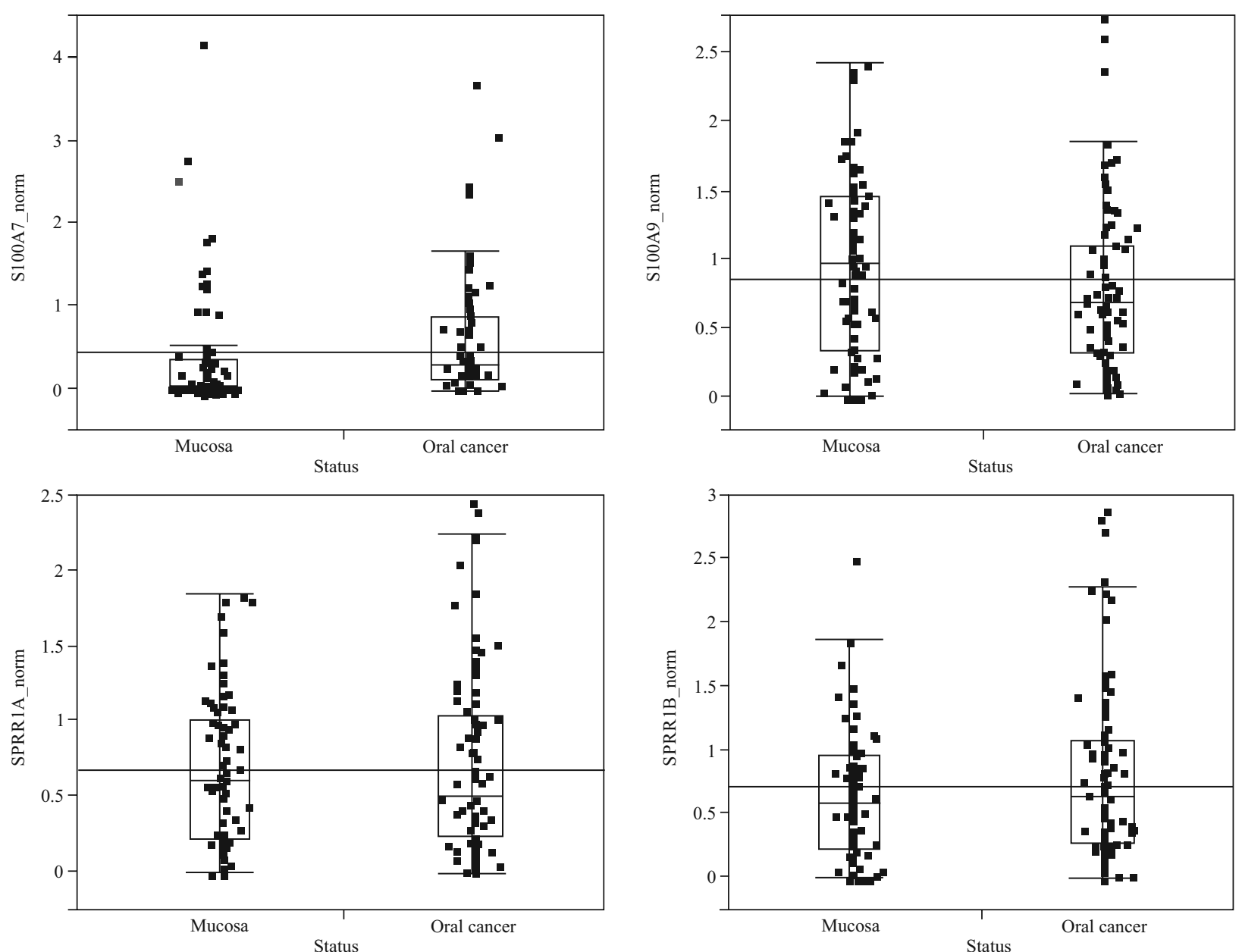

Figure 3. QPCR validation of S100A7 down-regulation in oral cancer. Y axis presents normalized gene expression values as measured by QPCR (arbitrary units). Differences are significant for $S 100 A 7$ ( $\mathrm{p}<0.05$, U Mann-Whitney test). Vertical line denotes grand mean in both groups

-normal difference within all analyzed EDC genes, based on the comparison between oral cavity SCCs to matching normal mucosa. There are numerous reports confirming S100 genes alterations in many tumors and some of the changes were suggested as markers of tumor progression [18]. The potential 
prognostic significance of S100 proteins was demonstrated by Albuquerque et al. who associated S100 immunohistochemical (IHC) expression with the histological grading in oral cancer [19] and stressed the relative heterogeneity related to the anatomical location of the tumor.

In our analysis we found significantly decreased expression of two S100 proteins: S100A1 and S100A4 in oral SCC. In previous studies $S 100 A 1$ gene expression, determined by microarray, QPCR and Western blot analyses, was shown to be relatively low in most normal tissues, however, it was up-regulated in kidney, ovarian and skin cancers [20]. Moreover, it may constitute a negative prognostic factor in some cancers - e.g. in patients with endometrioid subtype of ovarian and endometrial carcinoma - DeRycke et al. demonstrated in this setting a negative correlation between $S 100 A 1$ expression and relapse free survival time [20]. We also observed a relatively low S100A1 expression in normal oral mucosa, with even more reduced expression in oral tumors, suggesting tissue-specific differences of gene regulation.

S100A4 (metastatin) is known to be expressed in nervous system, where it is probably involved in neurite extension. It is over-expressed in many tumors, among them breast, lung, colorectal, gastric, prostate, ovarian, and pancreatic cancers as well as malignant melanoma [21]. It was demonstrated by Grigorian et al. that, by interaction with p53, S100A4 inhibits p53-mediated tumor suppression [22]. Combined aberrant expression of E-cadherin and S100A4, but $\operatorname{not} \beta$-catenin was associated with disease-free survival and overall survival in colorectal cancer patients [23]. This association could also be a mechanism of regulation of angiogenesis and tumor invasion, as shown in thyroid cancer [24]. S100A4 levels in pancreatic cancer were suggested to predict radioresistance [25]. Although originally $S 100 A 4$ role has been identified in metastatic breast cancer cells [26], it has been recently suggested that $S 100 A 4$ expression may be induced by the tumor microenvironment also in oral cancer [27].

There are published data available showing the possible association between S100 proteins and head and neck cancer. The altered expression of various S100 proteins was reported in primary and metastatic laryngeal carcinomas [28] and epithelial tumors of the skin [29]. S100A4 was confirmed to regulate E-cadherin expression in oral SCC cell lines [30], and its expression was correlated with invasion and metastasis [31]. It was shown to mediate epithelial-mesenchymal transition in HNSCC and was associated with stem-cell like phenotype (S100A4 knockdown reduced self-renewal and stemness of cells, while $S 100 A 4$ over-expression enhanced their stem cell properties) [32].
In head and neck cancer higher expression of S100A4 assessed by immunohistochemistry was reported by de Oliveira et al. [18] in patients with metastatic lymph nodes as compared with the non-metastasizing tumors. The expression of $S 100 A 4$ was analyzed parallel to endoglin, a protein probably regulated by S100A4, which also showed similar pattern of expression. In our study we observed a significant down-regulation of $S 100 A 4$ gene expression in oral primary SCC vs. matched adjacent normal tissue. Similarly to $S 100 A 1$ the expression of $S 100 A 4$ gene observed in our study on transcript level was lower in normal mucosa, when compared to other S100 genes. Its decrease in cancer requires further analysis to check if this is associated with functional effects. Interestingly, Oliveira et al. showed that lower expression of S100A4 in primary non-metastatic HNSCC samples was not associated with altered methylation status of its gene as compared to metastatic tissue [18].

Other S100 proteins found by us to be up-regulated in HNSCC samples have also been linked to cancer biology. $S 100 A 7$ (psoriasin) is a DNA damage-inducible gene [33], which is primarily involved in chemotaxis of inflammatory cells. It is up-regulated in breast, bladder and skin cancers [34]. According to Emberley et al. S100A7 protein expression correlates with aggressive course and overall survival in breast cancer [26]. An increased nuclear localization of S100A7 was associated with poorer prognosis in HNSCC [35]. Winter et al. assessed the transcript expression of $S 100 A 7$ in oral lesions and confirmed the up-regulation of this gene [36]. Our data support these observations and show $S 100 A 7$ to be the most significantly over-expressed gene within the EDC cluster with observed fold change 3.9 and 5.4 (by microarray and QPCR, respectively).

We have also noted a slight (1.5-fold change) but significant up-regulation of $S 100 A 11$ in cancer tissues. Anania et al. found that in papillary thyroid cancer an over-expression of $S 100 A 11$ may contribute to the malignant phenotype [37], moreover, the role of S100-positive dendritic cells in this context has been recently discussed [38]. In laryngeal cancer S100A11 gene was over-expressed both on mRNA and protein level and its expression was associated with a migratory capacity [39].

In the current study we observed a borderline significant up-regulation of $S 100 A 2$ and $S 100 A 3$ in comparison to normal mucosa (fold change 1.6 and 1.4 , respectively). The role of $S 100 A 2$ in carcinogenesis has been previously discussed [40]. In contrast to our data, S100A2 was down-regulated in oral cancer cell lines as identified by mRNA profiling analysis and proposed a potential marker of poor prognosis 
in early stage oral cancer since Tsai et al. observed significant correlation between reduced nuclear levels of S100A2 protein and shorter disease-free survival in early stage oral cancer patients [10]. Low protein level of S100A2 was associated with lymph node metastases in stage I-II oral cavity SCC [11]. A higher incidence of late cervical metastasis was observed in $S 100 A 2$ negative tumors than in those with $S 100 A 2$ positive cancers. Moreover, in multivariate analysis S100A2 expression was the only independent factor for late cervical metastases [11]. Low expression of this protein was associated with worse outcome in pancreatic cancer after adjuvant treatment [41].

An aberrant $S 100 A 3$ gene expression has not been previously reported for HNSCC. However, chromosomal amplifications involving $S 100 A 3$ were found in esophageal SCC after exposure to tobacco and betel quid [42]. Furthermore, S100A3 up-regulation was shown in colorectal [43] and bladder [44] cancers in addition to gastric cancer where $S 100 \mathrm{A3}$ over-expression correlated with tumor differentiation and TNM stage [45].

Attention should also be focused on S100A8/ /S100A9 (calprotectin), which is a toll-like receptor 4 agonist and is considered a proinflammatory and malignancy-stimulating factor [46]. S100A8/A9 molecules have been associated with epithelial-mesenchymal transition in breast carcinoma cells [47], as well as with the progression of colorectal carcinoma [48]. S100A8 expression has been analyzed in HNSCC and various degrees of premalignant lesions, as well as in serum of HNSCC patients [49]. In fact, S100A8/A9 has been regarded as a Janus-faced molecule in cancer therapy and carcinogenesis [50]. In the current study, we did not observe any differences on $S 100 A 8 / A 9$ transcript levels between cancer and normal oral tissues.

In our study not all S100 transcripts were analyzed. E.g., decreased expression of S100A6 (calcyclin) in oral SCC [51] was also previously described, that gene was confirmed to be regulated on the epigenetic level $[52,53]$. Another gene, S100A14 may regulate the invasiveness of HNSCC by influencing the expression of matrix metalloproteinases [54], it may interact with S100A16 [55].

Among other 1q21 genes we observed an up-regulation of $L C E 3 D$. This gene has not been previously reported to be of increased expression in cancer tissues. Another, previously not thoroughly analyzed gene is cingulin, also observed as up-regulated in the current study. It is a potentially important adhesion molecule, currently thought to be regulated by EphrinB signaling [56, 57].

Small proline-rich proteins, like SPRR2A were found to be among the most highly up-regulated genes in many non-squamous, stressed and remodeling barrier epithelia [58]. Based on these data we expected to see their aberrant expression in HNSCC. Although the SPRR gene cluster showed some degree of deregulation, the observed changes of expression were without statistical significance in our study. Despite being within the same 1q21 locus, SPRRs showed fewer differences between tumor and normal tissues than S100 genes. Therefore, we were not able to confirm in non-keratinizing oral carcinoma observations made in skin cancer by de Heller-Milev et al. (immunohistochemistry and Northern blotting) [59]. However, such a trend was observed in laryngeal cancer since we observed aberrant SPRR2G expression.

It has to be stressed that the proteins originating from the complex structure of skin layers and regulating its keratinization may behave differently in various contexts $[60,61]$. When we compared our data with the analysis of Hudson et al., who suggested that coordinated over-expression of EDC genes is a hallmark of skin squamous cell carcinoma, we reached similar global conclusions [15]. The most over-expressed gene in their study was $\$ 100 A 12$, and other eight S100 genes were over-expressed. Moreover, they found over-expression of five SPRR genes, involucrin and $L C E 3 D$ gene. For $L C E 3 D$ over-expression the results are in perfect agreement with our conclusion. The global over-expression of S100 genes is also in concordance with our observations, although our set of oral cancer patients provides strong suggestion that some S100 transcripts (S100A1/S100A4) may be down-regulated.

Thiel et al. attempted to analyze all differentially expressed proteins in oral squamous cell carcinoma by proteomic techniques (MALDI-TOF mass spectrometry) [62]. Significant expression and most notable differences were reported for involucrin and stratifin. The other authors also found differences in gene expression of stratifin and involucrin in addition to S100 calcium binding proteins [63]. However, we did not observe any differential expression of involucrin and stratifin transcripts in the current study.

At the moment it is not clear whether EDC genes are associated with any prognostic or predictive significance. When different biological parameters were analyzed in the context of clinical data in patients with oral tongue carcinoma stage I and II treated by intraoral excision only, it was found that mainly clinical parameters and E-cadherin expression were predictive of survival [64]. Other widely analyzed biological parameters like e.g. laminin $[65,66]$ did not reveal statistical significance.

In summary, coordinated over-expression of some epithelial differentiation complex genes, mainly from 
S100 family, seems to be a constitutive feature of head and neck cancer. The biological and clinical importance of these findings warrants further study.

\section{Acknowledgments}

The study was supported by Polish National Science Center grant no. N N401 423439 to A.M.

\section{References}

1. Kypriotou M, Huber M, Hohl D. The human epidermal differentiation complex: cornified envelope precursors, S100 proteins and the "fused genes" family. Exp Dermatol. 2012;21:643-649.

2. Kimchi ET, Posner MC, Park JO et al. Progression of Barrett's metaplasia to adenocarcinoma is associated with the suppression of the transcriptional programs of epidermal differentiation. Cancer Res. 2005;65:3146-3154.

3. Zucchini C, Biolchi A, Strippoli Pet et al. Expression profile of epidermal differentiation complex genes in normal and anal cancer cells. Int J Oncol. 2001;19:1133-1141.

4. Srikrishna G, Freeze HH. S100 protein family and tumorigenesis. Available at:http://atlasgeneticsoncology.org/Deep/ /S100ProtFamilyTumorID20092.html. Accessed June, 2014.

5. Salama I, Malone PS, Mihaimeed F, Jones JL. A review of the S100 proteins in cancer. Eur J Surg Oncol. 2008;34:357-364.

6. Sherbet GV. Metastasis promoter S100A4 is a potentially valuable molecular target for cancer therapy. Cancer Lett. 2009;280:15-30.

7. Mishra SK, Siddique HR, Saleem M. S100A4 calcium-binding protein is key player in tumor progression and metastasis: preclinical and clinical evidence. Cancer Metastasis Rev. 2012;31:163-172.

8. Kesting MR, Stoeckelhuber M, Kuppek A et al. Human $\beta$-defensins and psoriasin/S100A7 expression in salivary glands: anti-oncogenic molecules for potential therapeutic approaches. BioDrugs. 2012;26:33-42.

9. Jackson B, Tilli CM, Hardman MJ et al. Late cornified envelope family in differentiating epithelia - response to calcium and ultraviolet irradiation. $J$ Invest Dermatol. 2005;124:1062-1070.

10. Tsai ST, JinYT, Tsai WC et al. S100A2, a potential marker for early recurrence in early-stage oral cancer. Oral Oncol. 2005;41:349-357.

11. Suzuki F, Oridate N, Homma A et al. S100A2 expression as a predictive marker for late cervical metastasis in stage I and II invasive squamous cell carcinoma of the oral cavity. Oncol Rep. 2005;14:1493-1498.

12. Lauriola L, Michetti F, Maggiano N et al. Prognostic significance of the $\mathrm{Ca}(2+)$ binding protein S100A2 in laryngeal squamous-cell carcinoma. Int J Cancer. 2000;89:345-349.

13. Lohman FP, Medema JK, Gibbs S, Ponec M, van de Putte P, Backendorf C. Expression of the SPRR cornification genes is differentially affected by carcinogenic transformation. Exp Cell Res. 1997;231:141-148.

14. Lemaire F, Millon R, Young J et al. Differential expression profiling of head and neck squamous cell carcinoma (HNSCC). Br J Cancer. 2003;89:1940-1949.

15. Hudson LG, Gale JM, Padilla RS et al. Microarray analysis of cutaneous squamous cell carcinomas reveals enhanced expression of epidermal differentiation complex genes. Mol Carcinog. 2010;49:619-629.
16. Markowski J, Oczko-Wojciechowska M, Gierek T et al. Gene expression profile analysis in laryngeal cancer by high-density oligonucleotide microarrays.JPhysiol Pharmacol. 2009;60:57-63.

17. Jarzab J, Filipowska B, Zebracka J et al. Locus 1q21 Gene expression changes in atopic dermatitis skin lesions: deregulation of small proline-rich region 1A. Int Arch Allergy Immunol. 2010;151:28-37.

18. de Oliveira MV, de Carvalho Fraga CA, Barros LO et al. High expression of S100A4 and endoglinis associated with metastatic disease in head and neck squamous cell carcinoma. ClinExp Metastasis. 2014. May 6 [Epub ahead of print].

19. Albuquerque RLC, Miguel MCC, Costa ALL, Souza LB. Correlation of c-erbB-2 and S-100 expression with the malignancy grading and anatomical site in oral squamous cell carcinoma. Int J Exp Pathol. 2003;84:259-265.

20. DeRycke MS, Andersen JD, Harrington KM et al. S100A1 expression in ovarian and endometrial endometrioid carcinomas is a prognostic indicator of relapse-free survival. $\mathrm{Am}$ J Clin Pathol. 2009;132:846-856.

21. Sapkota D, Bruland O, Bøe OE et al. Expression profile of the S100 gene family members in oral squamous cell carcinomas. J Oral Pathol Med. 2008;37:607-615.

22. Grigorian M, Andresen S, Tulchinsky E et al. Tumor suppressor p53 protein is a new target for the metastasis-associated Mts1/S100A4 protein: functional consequences of their interaction. J Biol Chem. 2001;276:22 699-22 708.

23. Lee SJ, Choi SY, Kim WJ et al. Combined aberrant expression of E-cadherin and $\mathrm{S} 100 \mathrm{~A} 4$, but not $\beta$-catenin is associated with disease-free survival and overall survival in colorectal cancer patients. Diagn Pathol. 2013;8:99.

24. Jia W, Gao XJ, Zhang ZD, Yang ZX, Zhang G. S100A4 silencing suppresses proliferation, angiogenesis and invasion of thyroid cancer cells through downregulation of MMP-9 and VEGF. Eur Rev Med Pharmacol Sci. 2013;17:1495-1508.

25. Kozono S, Ohuchida K, Ohtsuka T et al. S100A4 mRNA expression level is a predictor of radioresistance of pancreatic cancer cells. Oncol Rep. 2013;30:1601-1608.

26. Emberley ED, Murphy LC, Watson PH. S100A7 and the progression of breast cancer. Breast Cancer Res. 2004;6:153-159.

27. Wetting HL, Hadler-Olsen E, Magnussen S et al. S100A4 expression in xenograft tumors of human carcinoma cell lines is induced by the tumor microenvironment. AmJ Pathol. 2011;178:2389-2396.

28. Nakashima T, Yano G, Hayashi I, Katsuta Y. Epithelial membrane antigen and S-100 protein-labeled cells in primary and metastatic laryngeal carcinomas. Head Neck. 1992;14:445-451.

29. Shrestha P, Muramatsu Y, Kudeken W et al. Localization of $\mathrm{Ca}(2+)$-binding $\mathrm{S} 100$ proteins in epithelial tumours of the skin. Virchows Arch. 1998;4324:53-59.

30. Moriyama-Kita M, Endo Y, Yonemura Y et al. S100A4 regulates E-cadherin expression in oral squamous cell carcinoma. Cancer Lett. 2005;230:211-218.

31. Moriyama-Kita M, Endo Y, Yonemura Y et al. Correlation of S100A4 expression with invasion and metastasis in oral squamous cell carcinoma. Oral Oncol. 2004;401:496-500.

32. Lo JF, Yu CC, Chiou SH et al. The epithelial-mesenchymal transition mediator S100A4 maintains cancer-initiating cells in head and neck cancers. Cancer Res. 2011;711:1912-1923.

33. Kennedy RD, Gorski JJ, Quinn JE et al. BRCA1 and c-Myc associate to transcriptionally repress psoriasin, a DNA damage-inducible gene. Cancer Res. 2005;65:10 265-10 272.

34. Tiveron RC, de Freitas LC, Figueiredo DL, Serafini LN, Mamede RC, Zago MA. Expression of calcium binding protein S100 A7 (psoriasin) in laryngeal carcinoma. Braz J Otorhinolaryngol. 2012;78:59-65. 
35. Tripathi SC, Matta A, Kaur J et al. Nuclear S100A7 is associated with poor prognosis in head and neck cancer. PLoS One. 2010;5:e11939.

36. Winter J, Pantelis A, Reich R et al. Risk estimation for a malignant transformation of oral lesions by S100A7 and Doc-1 gene expression. Cancer Invest. 2011;29:478-484.

37. Anania MC, Miranda C, Vizioli MG et al. S100A11 overexpression contributes to the malignant phenotype of papillary thyroid carcinoma. J Clin Endocrinol Metab. 2013;98:E1591-E1600.

38. Hilly O, Koren R, Raz R et al. The role of s100-positive dendritic cells in the prognosis of papillary thyroid carcinoma. Am J Clin Pathol. 2013;139:87-92.

39. Wang C, Zhang Z, Li L et al. S100A11 is a migration-related protein in laryngeal squamous cell carcinoma. Int J Med Sci. 2013;10:1552-1559.

40. Wolf S, Haase-Kohn C, Pietzsch J. S100A2 in cancerogenesis: a friend or a foe? Amino Acids. 2011;41:849-861.

41. Bachet JB, Maréchal R, Demetter P et al. S100A2 is a predictive biomarker of adjuvant therapy benefit in pancreatic adenocarcinoma. Eur J Cancer. 2013;49:2643-2653.

42. Chattopadhyay I, Singh A, Phukan R et al. Genome-wide analysis of chromosomal alterations in patients with esophageal squamous cell carcinoma exposed to tobacco and betel quid from high-risk area in India. Mutat Res. 2010;696:130-138.

43. Liu B, Sun WY, Zhi CY et al. Role of S100A3 in human colorectal cancer and the anticancer effect of cantharidinate. Exp Ther Med. 2013;6:1499-1503.

44. Yao R, Lopez-Beltran A, Maclennan GT, Montironi R, Eble JN, Cheng L. Expression of S100 protein family members in the pathogenesis of bladder tumors. Anticancer Res. 2007;27:3051-3058.

45. Liu J, Li X, Dong GL et al. In silico analysis and verification of $\mathrm{S} 100$ gene expression in gastric cancer. BMC Cancer. 2008;8:261.

46. Ehrchen JM, Sunderkötter C, Foell D, Vogl T, Roth J. The endogenous Toll-like receptor 4 agonist S100A8/S100A9 (calprotectin) as innate amplifier of infection, autoimmunity, and cancer. J Leukoc Biol. 2009;86:557-566.

47. Cormier K, Harquail J, Ouellette RJ, Tessier PA, Guerrette R, Robichaud GA. Intracellular expression of inflammatory proteins S100A8 and S100A9 leads to epithelial-mesenchymal transition and attenuated aggressivity of breast cancer cells. Anticancer Agents Med Chem. 2013;14:35-45.

48. Duan L, Wu R, Ye L et al. S100A8 and S100A9 are associated with colorectal carcinoma progression and contribute to colorectal carcinoma cell survival and migration via Wnt $/ \beta$-catenin pathway. PLoS One. 2013;8:e62092.

49. Driemel O, Escher N, Ernst G, Melle C, von Eggeling F. S100A8 cellular distribution in normal epithelium, hyperplasia, dysplasia and squamous cell carcinoma and its concentration in serum. Anal Quant Cytol Histol. 2010;32:219-224.

50. Ghavami S, Chitayat S, Hashemi M et al. S100A8/A9: a Janus-faced molecule in cancer therapy and tumorgenesis. Eur J Pharmacol. 2009;625:73-83.

51. Yang X, Wei K-J, Zhang L et al. Decreased expression of S100A6 in oral squamous cell carcinoma. Oncol Rep. 2010;24:479-488.
52. Leśniak W, Słomnicki ŁP, Kuźnicki J. Epigenetic control of the S100A6 (calcyclin) gene expression. J Invest Dermatol. 2007;127:2307-2314.

53. Leśniak W, Słomnicki ŁP, Filipek A. S100A6 - new facts and features. Biochem Biophys Res Commun. 2009;390: 1087-1092.

54. Sapkota D, Bruland O, Costea DE, Haugen H, Vasstrand EN, Ibrahim SO. S100A14 regulates the invasive potential of oral squamous cell carcinoma derived cell-lines in vitro by modulating expression of matrix metalloproteinases, MMP1 and MMP9. Eur J Cancer. 2011;47:600-610.

55. Sapkota D, Costea DE, Ibrahim SO, Johannessen AC, Bruland O. S100A14 Interacts with S100A16 and Regulates Its Expression in Human Cancer Cells. PLoS One. 2013;8:e76058.

56. Citi S, Amorosi A, Franconi F, Giotti A, Zampi G. Cingulin, a specific protein component of tight junctions, is expressed in normal and neoplastic human epithelial tissues. Am J Pathol. 1991;138:781-789.

57. Lee HS, Daar IO. EphrinB reverse signaling in cell-cell adhesion: is it just par for the course? Cell Adh Migr. 2009;3:250-255.

58. Demetris AJ, Specht S, Nozaki I et al. Small proline-rich proteins (SPRR) function as SH3 domain ligands, increase resistance to injury and are associated with epithelial-mesenchymal transition (EMT) in cholangiocytes. J Hepatol. 2008;48:276-288.

59. De Heller-Milev M, Huber M, Panizzon R, Hohl D. Expression of small proline rich proteins in neoplastic and inflammatory skin diseases. Br J Dermatol. 2000;143: 733-740.

60. Ellis T, Smyth I, Riley E et al. Overexpression of Sonic Hedgehog suppresses embryonic hair follicle morphogenesis. Dev Biol. 2003;263:203-215.

61. Niderla-Bielińska J, Moskalewski S. Involucrin, but not filaggrin and Kdap mRNA, expression is downregulated in 3-D cultures of intact rat hair bulbs after calcium stimulation. Folia Histochem Cytobiol. 2011;49:335-343.

62. Thiel UJE, Feltens R, Adryan B et al. Analysis of differentially expressed proteins in oral squamous cell carcinoma by MALDI-TOF MS. J Oral Pathol Med. 2011;40: 369-379.

63. Odani T, Ito D, Li MH et al. Gene expression profiles of oral leukoplakia and carcinoma: genome-wide comparison analysis using oligonucleotide microarray technology. Int $J$ Oncol. 2006;28:619-624.

64. Lim SC, Zhang S, Ishii G et al. Predictive markers for late cervical metastasis in stage I and II invasive squamous cell carcinoma of the oral tongue. Clin Cancer Res. 2004;10:166-172.

65. Shruthy R, Sharada P, Swaminathan U, Nagamalini B. Immunohistochemical expression of basement membrane laminin in histological grades of oral squamous cell carcinoma: A semiquantitative analysis. J Oral Maxillofac Pathol. 2013;17:185-189.

66. Osiecka BJ, Marciniak Z, Jeleń M, Bowszyc-Dmochowska M. Evaluation of laminin and cytokeratin-10 expression in squamous cell carcinoma of the skin. Folia Histochem Cytobiol. 1997;35:121-122. 